\section{Anterior segment ischemia: etiology, assessment, and management}

SL Pineles ${ }^{1}$, MY Chang ${ }^{2}$, EL Oltra ${ }^{3}$, MS Pihlblad ${ }^{4}$, JP Davila-Gonzalez ${ }^{1}$, TC Sauer ${ }^{5}$ and FG Velez ${ }^{1,6,7}$

\begin{abstract}
Anterior segment ischemia (ASI) is a potentially serious but rare complication of strabismus surgery. Among several risk factors, ASI occurs after strabismus surgery because of the nature of the anterior segment circulation. Disinsertion of rectus muscles leads to a decrease in the blood supply to the various anterior segment structures. We report a series of retrospective and prospective studies performed by our group focused on determining the risk of anterior segment ischemia following strabismus surgery, diagnosis, and modifications to surgical techniques to minimize the impact on anterior segment circulation. We found a significant decrease in postoperative anterior segment blood flow when operating vertical rectus muscles. Plication procedures preserve anterior segment circulation, and modifications to the technique allow the performance of adjustable sutures. Small adjustable selective procedures that spare the ciliary vessels have been demonstrated to be effective in patients with vertical and torsional diplopia. Ciliary sparing augmented adjustable transposition surgery decreases the risk of anterior segment ischemia while allowing management of potential postoperative alignment complications. Finally, ocular coherence tomography angiography is a valuable quantitative and qualitative technique to evaluate anterior segment ischemia. Strabismus surgeons should be aware of the risks of anterior segment ischemia when operating vertical rectus muscles. Modifications to standard surgical techniques allow surgeons to perform complex strabismus surgery in patients at risk for anterior segment ischemia.

Eye (2018) 32, 173-178; doi:10.1038/eye.2017.248; published online 17 November 2017

\section{Introduction}

Anterior segment ischemia (ASI) is a potentially serious but rare complication of strabismus surgery. ASI has been described in patients with various vascular disorders, such as carotid artery or ophthalmic artery obstruction, carotidcavernous fistula, leukemia, sickle cell disease, and others. ${ }^{1-3}$ First reported in $1957,{ }^{4}$ ASI due to strabismus surgery occurs in $1 / 13000$ to $1 / 30000$ cases. $^{3}$ ASI occurs after strabismus surgery because of the nature of the anterior segment circulation. Typically, there are two anterior ciliary arteries on each of the four rectus muscles, except for the lateral rectus muscle that only has one. The anterior ciliary vessels contribute to several vascular plexuses, including the episcleral limbal plexus, the intramuscular circulation within the ciliary body, and the major arterial circle in the iris root. ${ }^{5}$ When the rectus muscles are disinserted from the sclera, the overlying anterior ciliary vessels are also interrupted. Interruption of the ciliary vessels leads to a decrease in the blood supply to the various anterior segment vascular plexuses that they support. Clinically, this decrease in blood flow results in a delay or absence of iris vessel filling in the quadrant corresponding to the tenotomized muscle, when imaged using iris fluorescein angiography. ${ }^{6}$ Interestingly, the effect of tenotomizing horizontal rectus muscles is much less severe than the potential ischemia induced by tenotomizing vertical rectus muscles. ${ }^{7}$ Therefore, the risk of ASI is increased when strabismus surgery is performed on two or more muscles and when vertical muscles are involved. Other risk factors for ASI include advanced age, vascular disease, diabetes mellitus, hyperviscosity, hemoglobinopathies, and 360 degree scleral buckling surgery for retinal detachment. ${ }^{5}$
\end{abstract}

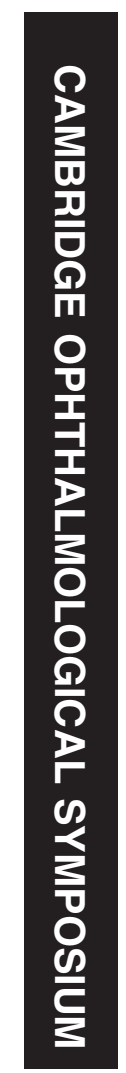

${ }^{1}$ Stein Eye Institute, University of California, Los Angeles, Los Angeles, CA, USA

${ }^{2}$ University of California, Davis, Sacramento, CA, USA

${ }^{3}$ Weill Cornell Medical College, New York, NY, USA

${ }^{4}$ Children's Hospital of Pittsburgh, University of Pittsburgh Medical Center, Pittsburgh, PA, USA

Ophthalmic Consultants of Boston-Tufts New England Eye Center, Boston, MA, USA

${ }^{6}$ Doheny Eye Institute, University of California, Los Angeles, Los Angeles, CA, USA

7Olive View UCLA-Medical Center, Sylmar, CA, USA

Correspondence:

FG Velez, Doris Stein Eye Institute, 200 Stein Plaza, Suite 3-519, UCLA, LOS Angeles, CA 90095 USA Tel: +1 310825 2872; Fax +1 3108250151 . E-mail: velez@jsei.ucla.edu

Received: 9 October 2017 Accepted: 9 October 2017 Published online: 17 November 2017 
ASI can present with a wide range of severity. In its mildest form, ASI presents with decreased iris perfusion and pupillary abnormalities. As severity increases, patients may demonstrate uveitis, keratopathy, hypotony and rarely cataract, corneal scar, and macular changes. ${ }^{7}$ Left untreated, the most severe cases can result in phthisis bulbi. ASI can be diagnosed based on clinical findings, but iris fluorescein angiography can also aid in making the diagnosis. Iris angiography in ASI typically reveals diffuse iris leakage or iris vascular filling defects in the quadrants adjacent to tenotomized muscles. ${ }^{8}$ Iris fluorescein angiography is difficult in heavily pigmented irises, and indocyanine green angiography has been used with better success in these patients. ${ }^{8}$ Most cases of ASI resolve after weeks to months as the anterior segment circulation collateralizes; however, patients may note persistent iris atrophy or pupil irregularity.

Various recommendations have been made to minimize the risk of ASI in patients undergoing strabismus surgery. It is generally recommended to avoid surgery on three or four rectus muscles simultaneously. Furthermore, additional techniques such as plication, ${ }^{9}$ vessel-sparing technique, ${ }^{5}$ and partial tenotomies ${ }^{10}$ have been reported. Additionally, some surgeons prefer to use fornix-based incisions and minimally invasive techniques to preserve the perilimbal episcleral vessels. ${ }^{11}$ Herein, we will describe recent work that aims to prevent ASI with novel surgical techniques and to report a new method of diagnosing ASI with the use of optical coherence tomography angiography (OCT-A).

\section{Anterior segment circulation in humans undergoing strabismus surgery}

Our group has worked on several modifications to current strabismus techniques in order to minimize ASI. For example, plication is an alternative tightening procedure to resection. Advantages of rectus muscle plication include reversibility, no risk of a lost or slipped muscle, and potentially less disruption of the anterior ciliary arteries. Studies in monkeys have shown that plication spares the anterior segment circulation. ${ }^{12-14}$

We performed a study in humans to confirm the effects of strabismus surgery on anterior segment circulation in adults. Iris angiograms were obtained in 14 patients undergoing strabismus surgery. Preoperative and 1-day postoperative iris angiograms were evaluated by a masked examiner.

Post-operative iris filling defects were seen in $66 \%$ of the patients who underwent tenotomies compared with $12.5 \%$ of the patients undergoing plication procedures.

All of the vertical tenotomized muscles showed postoperative iris filling defects. One plicated vertical muscle showed a postoperative filling defect; in this case, surgery on an adjacent horizontal muscle was performed simultaneously. Only one horizontal tenotomized muscle $(12.5 \%)$ showed a postoperative filling defect. No plicated horizontal muscles showed postoperative filling defects

From this study, we concluded that postoperative iris filling defects are more common following vertical rectus muscle surgery that involve muscle disinsertion. These findings are consistent with the well-known feature of anterior segment circulation being determined mainly by anterior ciliary vessels. The two long posterior ciliary arteries running beneath the medial and lateral rectus muscles supply between 20 and $30 \%$ of the anterior segment blood supply, and there are no superior or inferior long posterior ciliary arteries. ${ }^{15,16}$ This study also revealed that preserving the anterior ciliary vessel circulation may be important, especially when operating on the vertical rectus muscles. Plications and other ciliary vessel sparing procedures may be a safer alternative in patients at risk for ASI. ${ }^{9}$

\section{Adjustable plication procedures}

As described above, rectus muscle plication is a useful alternative to rectus muscle resection. ${ }^{12,17} \mathrm{~A}$ major advantage of a plication over a resection is the preservation of the anterior segment circulation as demonstrated above. ${ }^{9}$ Additional advantages of plication over resections include that the rectus muscle is not disinserted during the procedure, reducing the rare but feared complication of a 'lost muscle'. Plications are also less invasive with no irreversible removal of tendon with less surgical trauma, bleeding, and inflammation. The procedure is completely reversible early on, and this is another benefit. Similarly, adjustable sutures in strabismus surgery are another important tool for the strabismus surgeon. ${ }^{18,19}$ Postoperative adjustments give a second chance to the surgeon to obtain the desired postoperative alignment, ideally improving the success rate and reducing the re-operation rate.

We described a novel technique of an adjustable rectus muscle plication that gives multiple advantages over rectus muscle resection. These advantages include preservation of anterior segment circulation, possibility of postoperative adjustment, no muscle disinsertion, and less invasive with less surgical trauma and postoperative inflammation. ${ }^{20}$ In our study evaluating this technique, we found that the results concluded good short-term outcomes in their series with all patients having satisfactory outcomes without diplopia. The described technique of plicating the tendon on top of itself over an iris spatula did not result in any bulky mass or lump that has been a concern with plications. 
We also found that the adjustable plication technique is useful in reoperations on both vertical and horizontal rectus muscles. The technique gives the strabismus surgeon more options in different clinical situations as the preservation of the anterior segment circulation may allow the surgeon to add a third rectus muscle to a single procedure, or the ability to operate on a previously operated eye at risk for ASI. Additionally, this less invasive approach with less surgical trauma can be performed under topical anesthesia, unlike a more painful resection procedure that might not be as well tolerated.

The ability to avoid general anesthesia in certain patients can be a large benefit.

In conclusion, an adjustable rectus plication can be helpful in many difficult strabismus patients with many advantages over resection, particularly reducing the risk of ASI.

\section{Nystagmus surgery}

Patients with nystagmus and head turn are traditionally treated by recession and resection of the rectus muscles as described by Kestenbaum ${ }^{21}$ and Anderson. ${ }^{22}$ In some patients, especially those with multiplanar head posture requiring surgery on multiple rectus muscle, ${ }^{23}$ the risk for ASI is increased. The benefits of plication include a less invasive surgery, which is reversible immediately after surgery, and decreases the risk of ASI and intraoperatively lost muscles. ${ }^{9,17}$

We reported a case series of patients with nystagmus and anomalous head position (AHP) who underwent plication-only surgery of the horizontal rectus muscles. Rectus muscle plication was performed as previously reported with muscle body to sclera plication. ${ }^{17}$ Four patients were identified, ranging in age from 2 to 8 years. AHP had been measured and stable for at least 2 visits before surgery. Mean follow-up after surgery was 12 months (range 6-18 months).

Surgery was a combined medial rectus and contralateral lateral rectus plication ranging from 5 to $7.5 \mathrm{~mm}$ on the medial rectus and from 7 to $12 \mathrm{~mm}$ on the lateral rectus. Average preoperative horizontal AHP was 22.5 degrees and postoperative AHP 2.5 degrees (paired Student's $t$-test $P=0.034$ ) and only 1 patient required a second surgery. Preoperative horizontal heterotropia was a mean 4.0 diopters and postoperative 7.5 diopters (paired Student's $t$-test $P=0.455$ ). Binocular visual acuity improved in one patient and no patient had a loss in visual acuity. Three of four patients had resolution of AHP after the first surgery.

Treatment of AHP is a difficult problem and despite the numerous modifications made to the original Kestenbaum ${ }^{21}$ and Anderson ${ }^{22}$ procedures, reoperations and residual AHP or heterotropias are common. Parks and colleagues ${ }^{24}$ reported that 6 of 10 patients in one series had residual AHP. Similarly, Calhoun and colleagues $^{25}$ found 8 of 10 patients were not fully corrected. In our series, only one patient required a second surgery to resolve AHP. We therefore concluded that AHP can safely and effectively be treated with plication-only surgery.

\section{Adjustable small-incision selective tenotomy and plication for correction of incomitant vertical strabismus and torsion}

Perfusion of the anterior segment of the eye depends on the anterior ciliary vessels that travel within the four rectus muscles. Anterior segment ischemia, although rare, can complicate rectus muscle surgery. ${ }^{5}$ By avoiding transection of one or both anterior ciliary vessels within a rectus muscle, some surgeries potentially reduce the risk of this complication. ${ }^{9}$ We describe two surgical procedures to provide relative sparing of ciliary vessels while treating cyclovertical strabismus: selective tenotomy and plication of vertical rectus muscles. ${ }^{10}$ Surgery was performed on either the nasal or temporal fibers of vertical rectus muscles to correct horizontally incomitant vertical strabismus and/or cyclotorsion.

The following steps were performed for each surgery: after the vertical rectus muscle to undergo surgery was isolated, the nasal or temporal half of the muscle was secured with a 6-0 polyglactin 910 suture (in tenotomies, the muscle was sutured immediately posterior to the insertion; for plications, the suture was placed posterior to the insertion at a distance equal to the desired amount of plication); the suture was passed through the sclera adjacent to the insertion; the nasal or temporal fibers of the muscle were cut if a tenotomy was performed, or the suture advanced to the insertion if a plication was performed; and the suture was tied in an adjustable slip knot. Adjustment was performed on postoperative day 1 .

The mean age was 64 years (range $28-78$ years) and mean postoperative follow-up was 4.5 months (range 6 weeks to 10 months). Selective plications were performed on 4 patients, and selective tenotomies on 5 patients. All patients had horizontally incomitant vertical strabismus, and all except 1 (who underwent selective plication) had preoperative cyclotorsion.

Overall, horizontally incomitant vertical strabismus was successfully corrected in 8 of $9(89 \%)$ patients. Of the 8 patients with preoperative cyclotorsion, surgery successfully treated 4 (50\%). Of the 9 patients, 8 (89\%) reported resolution of diplopia postoperatively. All patients who experienced improvement in cyclotorsion had full correction postoperatively ( 0 degrees torsion). Therefore, the surgeries had an all-or-none effect on torsion. 
Our results show that selective tenotomy or plication of vertical rectus muscles can successfully correct horizontally incomitant vertical strabismus in most patients ( $89 \%$ in our series). These procedures have an allor-none effect on cyclotorsion. Selective tenotomy and plication for cyclotorsion may be less effective in patients with restriction from prior strabismus surgery or orbital disease. The procedures described in this study are variations of procedures described by others for correction of small hypertropias. ${ }^{12,26}$ Compared with other surgeries for cyclovertical strabismus ${ }^{27,28}$ selective tenotomy and plication have potential advantages including the ability to perform under topical anesthesia and use of adjustable sutures. Additionally, because plications do not involve transection of ciliary vessels, and selective tenotomies theoretically spare one of two ciliary arteries per rectus muscle, these procedures may reduce the risk of anterior segment ischemia compared with traditional rectus muscle surgery.

\section{Adjustable augmented ciliary vessel sparing transposition procedure}

Rectus muscle transposition procedures are indicated in patients with extraocular muscle palsies. ${ }^{29}$ The goal of the transposition procedure is to realign the eye by creating a vectorial force that balances the force of the ipsilateral antagonist muscle, and to enlarge the binocular field of vision. Potential complications of transposition procedures include ASI and induced vertical deviations. Ciliary vessel sparing, adjustable sutures, and augmentation sutures are some of the modifications that intend to decrease these complications. ${ }^{29-35}$

We introduced a novel modification of the transposition procedure augmented by advancement of the transposed muscles with ciliary vessel sparing and an adjustable technique. ${ }^{36}$ Our surgical technique includes a fornix incision followed by a muscle transposition using a 6-0 polyglactin suture that is secured on the muscle at a predetermined distance from the insertion (usually $5 \mathrm{~mm}$ posterior to the muscle insertion) incorporating one-third of the muscle width adjacent to the paralyzed muscle. The non-disinserted muscle is transposed by dragging the tendon-muscle complex towards the paralyzed muscle and anteriorly toward the paralyzed muscle insertion, reinserting the preplaced 6-0 polyglactin sutures adjacent to the insertion of the paralyzed muscle. The muscle is then attached to the sclera via an adjustable suture along the spiral of Tillaux.

Seven patients with abduction deficiency were retrospectively reported. There was a significant improvement in the angle of deviation near and distance. The mean preoperative deviation at distance decreased from $30.6 \pm 12.9$ prism diopters (range, $17-50 \mathrm{PD}$ ) to $10.6 \pm 8.8 \mathrm{PD}$ (range, $0-25 \mathrm{PD})$ postoperatively $(P=0.003)$. At near, the mean preoperative deviation improved from $19.3 \pm 11.4$ to $4.1 \pm 5.0 \mathrm{PD}$ postoperatively $(P=0.01)$. Postoperative adjustments were performed in three patients. Three patients required postoperative adjustment to correct an induced vertical deviation and an overcorrection. No patient showed clinical signs of anterior segment ischemia.

Rosenbaum ${ }^{29}$ foresaw the possibility of transposition surgery without disinserting the muscle, thus sparing all ciliary vessels. Full tendon rectus muscle transposition is believed to be more powerful than partial tendon transposition procedures; ${ }^{1-39}$ however, full tendon procedures do not preserve ciliary vessels. ${ }^{29-33}$ The posterior fixation suture increases the effect of the transposition procedures. ${ }^{34,35,37}$ However, the use of a augmentation suture precludes the use of adjustable sutures. Brooks et al $l^{40}$ introduced a modification of the partial transposition procedure by adding a resection of each transposed halve 4-8 $\mathrm{mm}$. This modification augmented the effect of the transposition without a posterior fixation suture. Nishida et $a l^{41}$ introduced a nonadjustable half tendon transposition procedure in which the muscle is never disinserted sparing all ciliary vessels. However, an adjustable transposition procedure has several advantages especially the correction of a postoperative induced vertical deviation and overcorrections. ${ }^{31,33,36,42-44}$

The current tendency among strabismus surgeons is to perform isolated rectus muscle transposition such as a superior rectus muscle augmented transposition to the lateral rectus muscle for an abduction deficiency. This procedure can be combined with simultaneous medial rectus muscle recession. Mehendale $e t a l^{45}$ have shown the surgical option of superior rectus transposition alone combined with medial rectus recession to have reduced a mean esotropia of $44 \Delta$ to $10 \Delta$ in patients with Duane syndrome and abducens palsy. In their series $20 \%$ of the patients with sixth nerve palsy developed postoperative vertical deviations. As described, the procedure does not allow adjustment of the transposed superior rectus muscle.

In conclusion, adjustable suture extraocular muscles transposition can be performed without muscle disinsertion preserving ciliary vessel circulation decreasing the overall risk of ASI and allowing simultaneous surgery on other ipsilateral rectus muscles as well as postoperative adjustment. Augmentation can be achieved by either resecting a portion of the transposed muscle or by simple advancement/plication of the transposed muscle. 
OCT-A for detection of anterior segment ischemia after strabismus surgery

Indocyanine green angiography (ICG-A) has been utilized to reveal iris filling defects to assess a patient's risk of ASI. ${ }^{46}$ However, ICG-A is limited by its invasive, timeconsuming nature and ability to image only unilaterally, as well as by potential side effects. OCT-A has been introduced and used to image iris vasculature in normal subjects and for iris tumors. ${ }^{47,48}$

Patients undergoing strabismus surgery underwent preoperative and postoperative iris ICG-A and OCT-A preoperatively and postoperatively. OCT-A revealed a significant decrease in the mean vessel density of the iris quadrant adjacent to the operated muscle postoperatively $(P<0.05)$. OCT-A was comparable to ICG-A for determining iris vessel filling defects. In addition, OCT-A gives quantitative vessel density values that can be compared pre- and post-operatively. This initial research has shown that OCTA can be a useful tool in the evaluation of ASI in patients undergoing strabismus surgery. Further research will determine the ability of OCTA to diagnose ASI in various clinical scenarios and levels of severity.

\section{Conflict of interest}

The authors declare no conflict of interest.

\section{Acknowledgements}

This study was supported by Research to Prevent Blindness unrestricted grant to SLP and FGV. Presented at the Cambridge Ophthalmological Society Symposium, Old Divinity School at St John's College, University of Cambridge, Cambridge, UK, 6-8 September 2017.

\section{References}

1 Bullock JD, Falter RT, Downing JE, Snyder HE. Ischemic ophthalmia secondary to an ophthalmic artery occlusion. Am J Ophthalmol 1972; 74(3): 486-493.

2 Coppeto JR, Wand M, Bear L, Sciarra R. Neovascular glaucoma and carotid artery obstructive disease. Am J Ophthalmol 1985; 99(5): 567-570.

3 Saunders RA, Bluestein EC, Wilson ME, Berland JE. Anterior segment ischemia after strabismus surgery. Surv Ophthalmol 1994; 38(5): 456-466.

4 Stucchi C, Bianchi G. Depigmentation en secteur de l'iris consecutive a des transplantaions musculaires. Ophthalmologica 1957; 133: 231-234.

5 McKeown CA. Anterior ciliary vessel sparing procedureIn: Rosenbaum AL, Santiago P (eds). Clinical Strabismus Management. Philadelphia: W.B. Saunders Company, 1999; pp 516-526.
6 Olver JM, Lee JP. The effects of strabismus surgery on anterior segment circulation. Eye (Lond) 1989; 3 (Pt): 3 318-326.

7 Olver JM, Lee JP. Recovery of anterior segment circulation after strabismus surgery in adult patients. Ophthalmology 1992; 99(3): 305-315.

8 Chan TK, Rosenbaum AL, Rao R, Schwartz SD, Santiago P, Thayer D. Indocyanine green angiography of the anterior segment in patients undergoing strabismus surgery. $\mathrm{Br} J$ Ophthalmol 2001; 85(2): 214-218.

9 Oltra EZ, Pineles SL, Demer JL, Quan AV, Velez FG. The effect of rectus muscle recession, resection and plication on anterior segment circulation in humans. $\mathrm{Br} J$ Ophthalmol 2015; 99(4): 556-560.

10 Chang MY, Pineles SL, Velez FG. Adjustable small-incision selective tenotomy and plication for correction of incomitant vertical strabismus and torsion. J AAPOS 2015; 19(5): 410-416.

11 Mojon DS. Comparison of a new, minimally invasive strabismus surgery technique with the usual limbal approach for rectus muscle recession and plication. $\mathrm{Br} J$ Ophthalmol 2007; 91(1): 76-82.

12 Leenheer RS, Wright KW. Mini-plication to treat small-angle strabismus: a minimally invasive procedure. J AAPOS 2012; 16: 327-330.

13 Park C, Min BM, Wright KW. Effect of a modified rectus tuck on anterior ciliary artery perfusion. Korean J Ophthalmol 1991; 5(1): 15-25.

14 Wright KW, Lanier AB. Effect of a modified rectus tuck on anterior segment circulation in monkeys. J Pediatr Ophthalmol Strabismus 1991; 28(2): 77-81.

15 Wilcox LM, Keough EM, Connally RJ, Hotte CE. The contribution of blood flow by the anterior ciliary arteries to the anterior segment in the primate eye. Exp Eye Res 1980; 30: 167-174.

16 Hayreh SS, Scott WE. Flourescein iris angiography. II. Disturbances in iris circulation following strabismus operation on the various recti. Arch Ophthalmol 1978; 96: 1390-1400.

17 Chaudhuri Z, Demer JL. Surgical outcomes following rectus muscle plication: a potentially reversible, vessel-sparing alternative to resection. JAMA Ophthalmol 2014; 132(5): 579-585.

18 Mireskandari K, Cotesta M, Schofield J, Kraft SP. Utility of adjustable sutures in primary strabismus surgery and reoperations. Ophthalmology 2012; 119(3): 629-633.

19 Nihalani BR, Hunter DG. Adjustable suture strabismus surgery. Eye 2011; 25(10): 1262-1276.

20 Velez FG, Demer JL, Pihlblad MS, Pineles SL. Rectus muscle plication using an adjustable suture technique. J AAPOS 2013; 17(5): 480-483.

21 Kestenbaum A. (New operation for nystagmsus). Bull Soci Bull Sociétés Ophtalmol Fr 1953; 6: 599-602.

22 Anderson JR. Causes and treatment of congenital eccentric nystagmus. Br J Ophthalmol 1953; 37(5): 267-281.

23 von Noorden GK, Jenkins RH, Rosenbaum AL. Horizontal transposition of the vertical rectus muscles for treatment of ocular torticollis. J Pediatr Ophthalmol Strabismus 1993; 30(1): 8-14.

24 Mitchell PR, Wheeler MB, Parks MM. Kestenbaum surgical procedure for torticollis secondary to congenital nystagmus. J Pediatr Ophthalmol Strabismus 1987; 24(2): 87-93.

25 Nelson LB, Ervin-Mulvey LD, Calhoun JH, Harley RD, Keisler MS. Surgical management for abnormal head 
position in nystagmus: the augmented modified Kestenbaum procedure. Br J Ophthalmol 1984; 68(11): 796-800.

26 Scott A. Graded rectus muscle tenotomy for small deviations. Proceedings of the Jampolsky Festschrift. San Francisco: The Smith-Kettlewell Eye Research Institute 2000; 215-216.

27 Harada M, Ito Y. Surgical correction of cyclotropia. Jpn J Ophthalmol 1964; 8: 88-96.

28 von Noorden GK, Jenkins RH, Chu MW. Horizontal transposition of the vertical rectus muscles for cyclotropia. Am J Ophthalmol 1996; 122(3): 325-330.

29 Rosenbaum AL. Costenbader Lecture: the efficacy of rectus muscle transposition surgery in esotropic Duane syndrome and VI nerve palsy. J AAPOS 2004; 8: 409-419.

30 Hummelsheim E. Uber Schnentransplantation am Auge. Ophthal Gesselschft 1907; 34: 248.

31 Carlson MR, Jampolsky A. An adjustable transposition procedure for abduction deficiencies. Am J Ophthalmol 1979; 87: 382-387.

32 Laby DM, Rosenbaum AL. Adjustable vertical rectus muscle transposition surgery. J Pediatr Ophthalmol Strabismus 1994; 31: 75-78.

33 Phamonvaechavan P, Anwar D, Guyton DL. Adjustable suture technique for enhanced transposition surgery for extraocular muscles. J AAPOS 2010; 14: 399-405.

34 Foster RS. Vertical muscle transposition augmented with lateral fixation. J AAPOS 1997; 1: 20-30.

35 Clark RA, Demer JL, Rosenbaum AL. Rectus extraocular muscle pulley displacement after surgical transposition and posterior fixation for treatment of paralytic strabismus. Am J Ophthalmol 2002; 133: 119-128.

36 Hendler K, Pineles SL, Demer JL, Yang D, Velez FG. Adjustable augmented rectus muscle transposition surgery with or without ciliary vessel sparing for abduction deficiencies. Strabismus 2014; 22(2): 74-80.

37 Struck MC. Augmented vertical rectus transposition surgery with single posterior fixation suture: modification of Foster technique. J AAPOS 2009; 13: 343-349.
38 Velez FG, Foster RS, Rosenbaum AL. Vertical rectus muscle augmented transposition in Duane syndrome. J AAPOS 2001; 5: 105-113.

39 Britt MT, Velez FG, Thacker N, Alcorn D, Foster RS, Rosenbaum AL et al. Partial rectus muscle-augmented transpositions in abduction deficiency. J AAPOS 2003; 7: 325-332.

40 Brooks SE, Olitsky SE, deB Ribeiro G. Augmented Hummelsheim procedure for paralytic strabismus. J Pediatr Ophthalmol Strabismus 2000; 37: 189-195.

41 Nishida Y, Inatomi A, Aoki Y, Hayashi O, Iwami T, Oda S et al. A muscle transposition procedure for abducens palsy, in which the halves of the vertical rectus muscle bellies are sutured onto the sclera. Jpn J Ophthalmol 2003; 47: 281-286.

42 Ruth AL, Velez FG, Rosenbaum AL. Management of vertical deviations after vertical rectus transposition surgery. J AAPOS 2009; 13: 16-19.

43 Fitzsimons R, Lee JP, Elston J. Treatment of sixth nerve palsy in adults with combined botulinum toxin chemodenervation and surgery. Ophthalmology 1988; 95: 1535-1542.

44 Rosenbaum AL, Kushner BJ, Kirschen D. Vertical rectus muscle transposition and botulinum toxin (Oculinum) to medial rectus for abducens palsy. Arch Ophthalmol 1989; 107: 820-823.

45 Mehendale RA, Dagi LR, Wu C, Ledoux D, Johnston S, Hunter DG. Superior rectus transposition and medial rectus recession for Duane syndrome and sixth nerve palsy. Arch Ophthalmol 2012; 130: 195-201.

46 Chan TK, Rosenbaum AL, Rao R, Schwartz SD, Santiago P, Thayer $\mathrm{D}$. Indocyanine green angiography of the anterior segment in patients undergoing strabismus surgery. $\mathrm{Br} \mathrm{J}$ Ophthalmol 2001; 86: 214-218.

47 Allegrini D, Montesano G, Pece A. Optical coherence tomography angiography in a normal iris. Ophthalmic Surg Lasers Retina 2016; 47: 1138-1142.

48 Skalet AH, Li Y, Lu CD, Jia Y, Lee B, Husvogt L et al. Optical coherence tomography angiography characteristics of iris melanocytic tumors. Ophthalmology 2017; 124: 197-204. 